\title{
Politique
}

\section{Les rapports politiques au sein du mouvement des femmes au Québec}

\section{Michèle Lamont}

Numéro 5, hiver 1984

Femmes et pouvoir

URI : https://id.erudit.org/iderudit/040446ar

DOI : https://doi.org/10.7202/040446ar

Aller au sommaire du numéro

Éditeur(s)

Société québécoise de science politique

ISSN

0711-608X (imprimé)

1918-6584 (numérique)

Découvrir la revue

Citer cet article

Lamont, M. (1984). Les rapports politiques au sein du mouvement des femmes au Québec. Politique, (5), 75-107. https://doi.org/10.7202/040446ar d'utilisation que vous pouvez consulter en ligne.

https://apropos.erudit.org/fr/usagers/politique-dutilisation/ 


\title{
Les rapports politiques au sein du mouvement des femmes au Québec
}

\author{
Michèle Lamont \\ Stanford University
}

La Fédération des Femmes du Québec regroupe 75000 membres. Les Cercles des Fermières en regroupent 74400 , et l'Association féminine d'Éducation et d'Action sociale, 35000. 25000 femmes participent aux activités du 8 mars $1981^{1}$. Les Centres de femmes passent de 4 qu'ils étaient en 1979, à 36 en 1983, déservant chacun une population moyenne de 200 usagères régulières ${ }^{2}$. Le Front commun contre la Pornographie regroupe 300000 membres, appuyés par 70 associations ${ }^{3}$. L'Institut Simone de Beauvoir (Université Concordia) organise en juillet 1982 un colloque international sur l'enseignement et la recherche relatifs aux femmes, réunissant 300 invités de 80 pays. La revue La Vie en Rose tire à 12000 exemplaires $^{4}$, Châtelaine à 295000, soit 40000 de plus que Actualité. Si le mouvement des femmes détient peu

1. Fédération professionnelle des journalistes du Québec, Thérèse, Janette, Hélène et les autres, ou la face cachée de l'information, Bilan du colloque "Les femmes et l'information", 1973,45 .

2. La Vie en Rose, mars 1983, 56.

3. Le Devoir, 21 mars 1983, 4.

4. Thérèse, Janette, Hélène et les autres, ou la face cachée de l'information, op. cit., 40; données de 1982 . 
de légitimité en tant qu'objet sociologiques, c'est sans doute en dépit de son importance en tant que phénomène social ${ }^{6}$.

L'objectif de cet article est de construire un premier portrait approximatif du champ féminin et féministe québécois ${ }^{7}$. Nous allons situer les uns par rapport aux autres des organismes intervenant dans la définition des nouvelles fonctions, positions et représentations sociales des femmes. On comparera ces organisations sur la base: 1) de l'influence qu'elles exercent sur le champ et 2) du degré de radicalisme de leurs revendications. Ensuite, on situera ces organisations à l'intérieur de sous-champs selon leur secteur d'activité. Les résultats permettront de positionner à l'intérieur d'un même tableau symbolisant le champ, des organismes aussi divers que les Cercles des Fermières, le Théâtre expérimental des Femmes, le Comité d'action féministe du Y.W.C.A., le Conseil du Statut de la Femme et les Éditions Remue-Ménage. ${ }^{8}$

5. «La sociologie des femmes est en voie de devenir une spécialité reconnue, mais aussi du point de vue de l'institution, un ghetto, une voie de garage, un cul de sac." Laurin-Frenette, N., "Présentation; les femmes dans la sociologie», Sociologie et Société, vol. 13, no. 2, octobre 1981, 8.

6. On peut évaluer au dixième de la population féminine celles qui interviennent directement dans le mouvement des femmes au Québec. Et ceci constitue un chiffre conservateur puisque, à elle seule, la Fédération des Femmes du Québec regroupait 300000 membres en 1976. Les femmes constituent donc un des secteurs de la population québécoise les plus mobilisés.

7. Le concept de champ renvoit à l'idée d'un ensemble de relations sociales structurées autour d'un enjeu commun aux agents. Ceux-ci sont liés par une reconnaissance de la légitimité de l'enjeu autour duquel ils organisent leurs activités. Nous identifions l'enjeu du champ considéré ici à la définition des nouvelles fonctions, positions et représentations sociales des femmes. (Au sujet du concept de champ, élaboré par Pierre Bourdieu et l'équipe du Centre de sociologie européenne (E.H.E.S.S., Paris), voir Caro, J.Y., As if, tout se passe comme si..., thèse de doctorat d'État, Université de Paris 9, 1980).

Pour quiconque se situant à l'intérieur du champ, et y investissant intensément, les différences importantes qui distinguent les organismes féministes des organismes féminins interdisent de les classer dans un même champ. La proximité sociale aux enjeux accentue les différences. Partant d'un point de vue externe, nous faisons pour le moment abstractions de ces différences concrètes pour mettre l'emphase sur les effets sociaux de longue durée que sous-tendent les revendications du mouvement des femmes.

8. Certaines recherches ont analysé l'évolution du mouvement des femmes aau Québec (Voir entre autres Lanctôt, M., La naissance du mouvement des femmes à Montréal, 1969-76, thèse de maîtrise, Université du Québec à Montréal, 1981). L'originalité de 
L'objectif de cet article est aussi de reconstruire les relations politiques du champ. On considèrera 1) la position hiérarchique des organisations dans le champ selon l'influence qu'elles y exercent, et 2) leur position à l'intérieur du conflit principal qui anime le champ, lequel est identifié à une opposition entre des positions radicales ou réformistes à l'égard des revendications du mouvement des femmes. On construira une structure hiérarchique et une structure polaire (organisée autour du conflit principal) après avoir précisé les principes de structuration du champ9.

Il n'est pas de tradition de soumettre le mouvement des femmes à une analyse politique. La plupart des recherches sur le champ partent d'une perspective «interniste». A contrario, nous transposons au mouvement un cadre d'analyse qui ne tient pas sa légitimité de critères propres au champ féminin/féministe. Sa finalité est d'éclairer une nouvelle dimension du phénomène, généralement négligée. La pertinence d'une préoccupation si «scolaire» doit être reconnue si le mouvement des femmes devient un objet de recherche légitime - donc susceptible d'être soumis à divers types d'analyse... ${ }^{10}$.

notre contribution consiste à saisir le mouvement à partir des relations politiques entre ses parties.

9. Mettant l'accent sur les structures politiques, sur l'ossature du champ, nous serons forcés de négliger l'activité subjective - et la conscience de cette activité - par laquelle les agents élaborent ces structures. D'où le fait que les militantes auront des difficultés à reconnaître leur pratique dans ies pages qui suivent; on ne peut à la fois exercer une activité et être conscient du processus par lequel elle est produite, de la même manière qu'une socialisation ne peut avoir d'efficace si elle est vécue explicitement par les agents comme socialisation. Il doit y avoir une croyance - donc une absence de rupture - dans les finalités de l'activité pour que celle-ci puisse être exécutée.

La dimension dynamique du champ, le processus par lequel il est produit, ne pourront être considérés, compte tenu de nos choix de recherche. D'autres études analysent cet aspect du mouvement des femmes. (Voir Lamoureux, D., «Les difficultés d'émergence du mouvement autonome des femmes au Québec, thèse de doctorat de $3^{c}$ » cycle, E.H.E.S.S., Paris, 1982.)

10. Certains pourraient questionner l'existence même d'un champ féminin/féministe, et par extension, la pertinence du type d'analyse opérée ici. À ceux-ci, faisons remarquer qu'il existe un bon nombre d'associations formées autour des enjeux propres au champ, et que ces associations sont la preuve vivante de l'existence de ce champ. 
Nous considérons donc les organisations du champ à titre d'unités d'analyse: il s'agit là d'unités sociales identifiables, contenant chacunes des caractéristiques particulières. Leur mise en rapport permettra de dégager les caractéristiques générales du champ ${ }^{11}$.

Compte tenu du nombre considérable d'organisations intervenant dans le champ féminin/féministe, nous avons dû délimiter notre objet. Ne sont considérés 1) que des organismes nationaux, ou des organismes régionaux, montréalais en particulier, cherchant à avoir un rayonnement national ${ }^{12}, 2$ ) que des organismes francophones ou bilingues, les organismes anglophones se définissant surtout en fonction du champ féminin/féministe canadien ${ }^{13}$. À l'intérieur de ces frontières, nous n'avons retenu qu'un certain nombre d'organismes dont la prise en compte est essentielle à la compréhension du champ ${ }^{14}$. Nous avons cherché à rendre compte de la diversité du champ en retenant des organismes de divers types (revues, associations féminines, organismes gouvernementaux, services, fronts de revendication). Des contraintes d'espace nous

11. Il ne s'agit pas de faire le portrait exhaustif de ces organisations. Les organisations se positionnent structurellement de manière relationnelle. Leurs caractéristiques ne prennent leur signification sociale que par opposition, les propriétés des unes éclairant les propriétés des autres. (Voir entre autres Stone, G.P., "Appearance and the Self» in Rose, A.M., Human Behavior and Social Processes, Boston, Houghton Mifflin, 1962). D'où le fait qu'une comparaison des organisations sera à la base de la méthode adoptée pour construire les structures politiques du champ.

12. Ont été exclus les organismes fédéraux intervenant dans le champ québécois, tel le Conseil consultatif de la situation de la femme. Leur prise en compte aurait nécessité une analyse de leur position au sein du champ féminin/féministe canadien.

13. La distance sociale entre les organismes francophones et les organismes anglophones se manifeste dans le fait que les militantes francophones sont très rarement en mesure de décrire les organismes anglophones. Ceci devient moins vrai à mesure que l'on s'élève dans la structure hiérarchique du champ, les agents y occupant des positions dominantes parvenant plus fréquemment à une perception synthétique du mouvement des femmes.

14. L'échantillon a été construit à partir de l'Annuaire des Femmes de Montréal, Éditions Communiqu'elles, C.I.R.F., 1982. 


\section{ont forcé à exclure arbitrairement de l'échantillon des groupes dont l'analyse aurait éclairé des aspects importants du champs ${ }^{15}$. Notre matériel d'analyse consiste en une série d'entrevues effectuées auprès d'informatrices ${ }^{16}$ et auprès de femmes impliquées officiellement dans diverses organisations ${ }^{17}$. À ces dernières, nous}

15. Dans une version antérieure de ce texte, nous avions positionné des organismes tels Les Éditions de la Pleine Lune, la mensuelle lesbienne " $\ddot{C} a$ s'attrape», le collectif féministe-chrétien "L'Autre parole», la revue Amazones d'Hier, Lesbiennes d'Aujourd'bui, le Carrefour des associations des familles monoparentales du Québec, le mouvement contre le viol, le Groupe interdisciplinaire pour l'enseignement et la recherche sur la condition féminine (U.Q.A.M.), l'Institut Simone de Beauvoir, Relais-Femmes et la Ligue des Femmes du Québec. Si ces organismes ne sont pas positionnés spécifiquement dans cette version de l'article, leur prise en compte a influencé notre analyse générale du champ.

Certains s'objecteront à ce que le terme «organisation» soit utilisé pour désigner tant des organismes gouvernementaux structurés, que des groupes de femmes fonctionnant sous forme de collectif. C'est par mesure d'économie que nous avons opté pour cette appellation, en dépit du fait qu'elle ne rende pas convenablement compte de la diversité des institutions regroupées dans le champ.

16. Sont considérés comme informatrices des femmes qui, de par leur profession, ont un accès privilégié à l'information, et sont en mesure de tracer un portrait global du mouvement des femmes. Nous avons rencontré: Christine Corbeil, Nancy Guberman et Diane Lamoureux (U.Q.A.M.), Nicole Laurin-Frenette (Université de Montréal), Danielle Levasseur (Radio-Canada), Francine Montpetit (Chatelaine), et Anne Richer (Journal La Presse).

17. Nous présentons ici la liste des interviewées, puisqu'il s'agit là de notre principale source d'information. Nous avons rencontré: Claudette Bastien (Comité d'action politique des femmes du Parti Québécois), Liliane Blanc (Fédération des Femmes du Québec), Ginette Boyer (L'Autre parole), Nicole Brossard (écrivaine, anciennement des Têtes de Pioche), Ariane Brunet (pour le collectif Amazones d'Hier, Lesbiennes d'Aujourd'bui, Lise Champagne (Au Bas de l'échelle), Marie-Josée Corriveau (Front-Femmes du Sommet populaire, Action-Travail des Femmes, anciennement de Des Luttes et des Rires de Femmes), Léa Cousineau (Relais-Femmes, conseil d'administration du Conseil du Statut de la Femme, anciennement du cabinet de la Ministre déléguée à la condition féminine), Louise Dupré (Éditions Remue-Ménage), Ariane Emond (La Vie en Rose, anciennement du Comité de Lutte pour l'Avortement et la Contraception libres et gratuits), Diane Gagné (Carrefour des associations des familles monoparentales du Québec, Comité d'action politique des femmes du Parti Québécois), Ann Gertler (Voice of Women), Isabelle Grémy (Groupe interdisciplinaire pour l'enseignement et la recherche sur la condition féminine, U.Q.A.M.), Michèle Jean (historienne, anciennement des Têtes de Pioche), Louise Joly (Association féminine pour l'Éducation et l'Action sociale), Danielle Hébert (Comité de la condition féminine, Conseil central de Montréal, C.S.N.), Lise Moisan (Comité d'action féministe, Y.W.C.A.), Francine Pelletier ( $L a$ Vie en Rose, anciennement du Comité de Lutte pour l'Avortement et la Contraception libres et gratuits), Anne-Hélène Perreault (Centre d'information et de référence des femmes), Marie Tremblay (Cercles des Fermières), Louise 
avons demandé de tracer un portrait global du mouvement des femmes d'y situer leur organisation, d'en décrire les activités et les objectifs etc... Nous leur avons également demandé de décrire d'autres organisations, d'en évaluer l'influence et le degré de proximité à l'égard de leur propre organisation, en ce qui a trait aux types de revendications soutenues, aux objectifs, aux moyens d'intervention. Nous avons ainsi cherché à reconstituer en partie l'état inter-subjectif du champ, c'est-à-dire le champ tel qu'il existe au niveau de l'inter-représentation des agents ${ }^{18}$. Simultanément, nous accumulions un ensemble d'informations ponctuelles qui allaient servir à la reconstitution de l'état objectif du champ (coopération inter-organismes, budget, membership etc.). Nous complétions ces informations par le dépouillement de divers documents produits par les organisations (bulletins d'information, pamphlets, mémoires etc. $)^{19}$.

Dans un premier temps, on présentera la méthode adoptée pour construire le champ, ainsi qu'un tableau synthétisant les résultats de l'analyse. Dans un deuxième temps, on décrira ce tableau, justifiant le positionnement des diverses organisations.

\footnotetext{
Vandelac (U.Q.A.M., anciennement du Centre des Femmes), et Andrée Yannacopoulo (anciennement du Regroupement des Femmes du Québec). Ces interviews, ainsi que celles réalisées auprès d'informatrices, ont été effectuées à Montréal en juin 1983. Il s'agissait d'entrevues semi-directives d'une heure environ. Une demi-douzaine de ces entrevues furent réalisées par téléphone, en une durée plus courte.

18. Les répondantes sont considérées ici comme incarnation d'un segment du champ; ayant été suffisamment socialisées dans les institutions du champ pour y occuper des positions officielles leur discours est une objectification de certaines caractéristiques particulières de ces institutions.

19. Il faut insister ici sur le fait que le positionnement des organismes dans le champ est établi strictement sur la base du matériel récolté au cours des entrevues et au sein des documents consultés. Les résultats pourront être discutés et revus à partir d'informations dont nous n'avons pu tenir compte ici.
} 


\section{PRINCIPES DE STRUCTURATION DU CHAMP}

Le tableau suivant (voir p. 87) symbolise le champ féminin et féministe québécois. Les organisations y sont situées horizontalement et verticalement les unes par rapport aux autres. Elles sont également regroupées à l'intérieur de cercles représentant divers sous-champs du champ féminin/féministe.

L'influence exercée par les organisations sur le champ est le principe de structuration vertical de celui-ci. Elle détermine la position de ces organisations à l'intérieur de la structure hiérarchique du champ. Le degré de radicalisme de leurs revendications agit comme principe de structuration horizontale du champ. Nous allons maintenant définir plus précisément le sens attribué à ces critères, ainsi que la méthode utilisée pour positionner les organisations.

Par «influence exercée par les organisations sur le champ», nous entendons leur capacité de déterminer les autres organisations qui constituent le champ, tant dans leurs caractéristiques que dans leur action ${ }^{20}$. Parmi de multiples formes, cette influence se manifeste entre autres par la capacité des organisations de faire reconnaître leur définition de l'enjeu comme étant légitime (obligeant ainsi les autres organisations à tenir compte de leur présence dans l'orientation de leurs activités), par leur capacité d'organisation, et par extension, par leur capacité d'obtenir leurs revendications (ce qui augmente leur visibilité et transforme les conditions de visibilité des autres organisations ${ }^{21}$.

20. Divers types d'intervention peuvent être à l'origine de cette influence. Les organisations peuvent exercer une influence directe de manière à transformer l'action des autres organisations, créer des transformations dans le champ amenant les autres organisations à se redéfinir, ou créer hors du champ des transformations qui affectent ces organisations.

21. Cette proposition sous-tend l'hypothèse selon laquelle les organismes auront d'autant plus d'influence dans le champ qu'elles ont une grande proximité à la culture et aux institutions socialement dominantes (cette proximité leur permettant d'obtenir plus facilement gain de cause pour leurs revendications). Leur capacité d'influencer serait explicable par le fait que cette proximité sociale est la condition d'élaboration d'alliances qui affectent les rapports de force dans le champ. 
Mesurer l'influence exercée par les organisations sur le champ est nécessairement une opération difficile ${ }^{22}$. Il faut adopter une méthode d'évaluation qui puisse rendre compte de l'influence globale comparée des organisations, indépendamment de ce qu'en dit tel agent particulier. À cet égard, les bases objectives sur lesquelles repose l'influence peuvent être de quelque utilité.

Nous assumons que l'exercice de l'influence a comme condition le contrôle de bases objectives agissant comme support à l'action des organisations ${ }^{23}$. Ces bases peuvent être mobilisées pour orienter directement ou indirectement les autres organisations: il s'agit de ressources institutionnelles ou financières, de savoir-faire, de prestige etc. ${ }^{24}$.

Pour mesurer empiriquement l'influence des organisations, il faudrait isoler des indicateurs pour chaque base objective d'influence (base économique, institutionnelle, symbolique...) en tenant compte de la conjoncture où elles sont mobilisées dans l'exercice de l'influence. Cette opération s'avère impossible, d'abord parce

Cette hypothèse ne fera pas directement l'objet d'une vérification dans ce texte. Cependant, les résultats de notre analyse peuvent fournir des indications relatives à la validité de cette hypothèse.

22. Au sujet de certaines difficultés, voir Dahl, R.A., Who Governs?, New Haven and London, Yale University Press, 1961, ch. 24. Toutes les organisations ne sont pas affectées dans une même proportion par une influence donnée. Toutes ne sont pas affectées par une même influence. L'évaluation de l'influence d'une organisation donnée variera d'un agent à l'autre, selon le degré auquel ils sont affectés par celle-ci.

23. Il faudrait introduire ici une discussion du concept d'influence. Des contraintes d'espace nous forcent à ne présenter que les éléments essentiels à la compréhension de l'analyse qui suit.

Pour une définition du pouvoir - et par extension de l'influence - à partir des bases (ressources) sur lesquelles il repose, et des relations de dépendance qui en résultent voir Emerson, R. «Power-Dependency Relations" American Sociological Review, vol. 27, No. 1, 1962, 31-41.

24. L'influence sous-tendue par ces bases varie en fonction 1) du besoin qu'en ont les autres agents pour réaliser leurs intérêts propres, et 2 ) de la rareté de ces bases, c'est-à-dire de la possibilité pour les autres agents d'y avoir accès par un intermédiaire autre. Ces deux dernières variables rendent compte de la conjoncture où l'influence peut être exercée, tandis que les bases objectives d'influence renvoient au potentiel d'influence objectivement exerçable par les agents. 
qu'il faudrait analyser un nombre infini de conjonctures, et ensuite parce que certaines bases objectives d'influence ne peuvent être mesurées convenablement à partir d'un seul indicateur ${ }^{25}$. Ceci étant posé, nous sommes forçés de construire la structure du champ à partir d'une approximation de l'influence potentiellement exerçable par les organisations, compte tenu des bases objectives dont elles disposent.

Pour chacune des organisations retenues, on identifiera leurs principales bases objectives et comparera l'influence qu'elles soustendent potentiellement en considérant ce qu'en disent globalement les répondantes ( «reputational power»), et ce qu'en transmettent les quelques indicateurs empiriques disponibles ${ }^{26}$. Cinq bases objectives seront prises en compte: les bases économique (ressources financières), organisationnelle (infra-structure administrative, degré d'institutionnalisation des organisations), les bases scientifique (expertise), symbolique (prestige) et sociale (volume du membership) ${ }^{27}$. On tient également compte des alliances institutionnelles (syndicats, universités, autres institutions du champ), celles-ci pouvant à l'occasion être mobilisées pour augmenter l'influence des organismes. La position hiérarchique relative des organisations dans le champ correspondra à l'importance relative de leurs bases objectives d'influence (étant posé que leur influence potentielle

25. C'est le cas notamment des bases symboliques d'influence: le prestige accordé à un agent varie selon les champs considérés et les critères de légitimité qui y dominent. Pour évaluer précisément le prestige détenu par un seul agent, il faudrait donc reconstruire entièrement le champ symbolique où il prend place, $y$ identifier les critères de légitimité dominants, évaluer le degré auquel il répond à ces critères, comparativement aux autres agents du champ. À ce sujet, voir Bourdieu, P., "Le marché des biens symboliques", L'Année sociologique, Paris, Presses Universitaires de France, 1972. Pour une illustration de construction d'un champ symbolique, voir Kaduschin, C., The American Intellectual Elite, Boston, Little Browne, 1974.

26. Les variables conjonctuelles déterminant le degré d'influence exercé ne seront considérées qu'occasionnellement, lorsqu'il sera possible d'identifier précisément des relations de dépendance inégale entre institutions.

27. Cette typologie des bases objectives n'est introduite ici qu'à titre d'instrument heuristique stratégique. Pour une discussion de ces bases, voir Claval, P., Espace et Pouvoir, Paris, Presses Universitaires de France, 1978. 
varie en fonction des bases objectives de pouvoir contrôlées). Ainsi les institutions occupant les positions les plus élevées sont celles qui exercent l'influence potentiellement la plus importante dans le champ.

Afin de situer les organismes dans un même tableau, il fallait adopter comme point de départ une base de pouvoir qui soit un dénominateur commun à la majorité des organisations. Une forte proportion des organismes considérés sont des associations, donc des institutions dont l'influence repose sur la force du nombre. Les bases sociales d'influence (membership) serviront donc de dénominateur commun. Dans un premier temps, nous avons situé hiérarchiquement les organisations en fonction de leur base sociale pour ensuite réajuster leur position en considérant les autres bases objectives dont elles disposent ${ }^{28}$. D'où le fait que dans le tableau, nous ayons mis en ordonnée une échelle logarythmique représentant le volume des groupes, malgré qu'il ne s'agisse pas du seul indicateur de biérarchisation utilisé.

Le principe de structuration horizontale du champ est le degré de radicalisme des revendications portées par les organisations ${ }^{29}$. Ce critère a été retenu car il revenait constamment comme élément de différenciation des organisations (radicalisme vs réformisme) dans la description du champ produite par les répondantes.

Il fallait d'abord identifier le degré de radicalisme des diverses revendications. Voulant éviter d'intervenir arbitrairement dans le débat, nous avons procédé par la méthode suivante: nous avons

28. C'est ainsi que le Conseil du Statut de la Femme occupe une position dominante dans le champ, malgré qu'il ne dispose d'aucun membership: l'importance de ses ressources financières et institutionnelles justifie sa position.

29. On considère non seulement les revendications des organisations, mais aussi la problématique générale qui sous-tend leur action, puisque l'intervention de certains groupes n'est pas centrée autour de revendications. Ces problématiques sont les suivantes: critique du patriarcat, autonomie des femmes, égalitarisme et modernisation des rôles traditionnels des femmes. 
constitué un inventaire des principales revendications portées par le mouvement des femmes depuis les 15 dernières années. Nous avons ensuite demandé aux répondantes de les situer dans un axe radicalisme/réformisme. Nous obtenions alors un positionnement des revendications allant de la critique de l'hétéro-société à la réforme du droit de la famille ${ }^{30}$. Le spectre de polarisation des revendications obtenu est présenté au sommet du tableau.

Pour situer les organisations dans le spectre de polarisation des revendications, nous avons d'abord demandé aux interviewées de décrire le type d'action mené par les diverses institutions. Ensuite, à partir des résultats d'entrevues et des documents produits par les organismes, nous avons identifié précisément leurs objectifs et revendications, et sur cette base, avons corrigé leur positionnement polaire. Les organisations prennent place horizontalement dans le tableau en fonction des principales revendications qu'elles portent ${ }^{31}$.

Après avoir situé les institutions dans le champ, nous leur avons superposé un certain nombre de sous-champs symbolisant l'intersection du champ féminin/féministe et de champs autres. Ces sous-champs (ou secteurs d'activité) sont formés autour de la

30. Dans certains cas, il était difficile d'obtenir un positionnement précis. Nous avons alors appliqué le principe de positionnement suivant: Nous évaluions dans quelle mesure les revendications soutenaient la transformation des structures sociales à l'origine des rapports de domination et d'inégalités hommes/femmes (rapport de sexe), ou au contraire, cherchaient à en corriger les effets sociaux temporels. Nous considérions également l'objet des revendications, selon qu'elles portaient sur la modernisation des fonctions sociales traditionnelles des femmes (maternité, éducation, travail domestique), ou sur la mise en place de nouvelles fonctions (accès au marché du travail, transformation des rôles sexuels), supposant que celles-ci sont plus radicales que celles-là puisqui elles affectent directement la position sociale des femmes comme groupe, plutôt que les conditions de reproduction de leurs anciennes fonctions. (L'hypothèse selon laquelle le mouvement des femmes est en partie un mouvement de modernisation est empruntée à Diane Lamoureux (op. cit.).)

31. Les organisations sont positionnées en fonction des descriptions obtenues en juin 1983. Ces positionnements correspondent en partie aux états antécédents des rapports politiques du champ, dans la mesure où la structure actuelle est produite en partie par les rapports antécédents entre organisations. 
rencontre des enjeux du champ féminin/féministe et d'enjeux autres (enjeu culturel: production de l'univers symbolique global; enjeu politico-administratif: allocation des ressources...). La prise en compte des secteurs d'activité veut traduire le fait que toutes les organisations (et par extension, le champ lui-même) fonctionne en interaction avec d'autres espaces sociaux; on verra que l'interaction des enjeux externes détermine en partie les caractéristiques des organisations, ainsi que leur position.

Seuls les secteurs d'activité où plusieurs organisations interviennent ont été considérés ${ }^{32}$. Il s'agit des sous-champs politicoadministratif, de la gauche, des services, du sous-champ culturel ${ }^{33}$. Ils sont représentés dans le tableau par des cercles (voir légende). Les objectifs et moyens d'action des organismes ont servi pour déterminer quels sont les secteurs d'activité dans lesquels ils prennent place. On décrit ces secteurs d'activité au moment où il est question des organisations qu'ils regroupent.

\section{DESCRIPTION DU CHAMP}

Nous allons maintenant décrire le champ en justifiant le positionnement des diverses organisations, ainsi que celui des sous-champs. Pour des raisons d'économie d'espace nous ne présenterons ici que les éléments essentiels à la compréhension du

32. Les organisations intervenant dans plusieurs sous-champs sont situées à l'intersection de ceux-ci. La position hiérarchique et polaire de certaines organisations ne permet pas toujours de les situer aux intersections. Elles sont alors intégrées au souschamp où leur intervention semble produire l'effet social le plus important. C'est le cas notamment de La Vie en Rose qui intervient tant dans le sous-champ culturel que dans le sous-champ de la gauche. Les Cercles des Fermières pour leur part interviennent à la fois dans le sous-champ politico-administratif et dans le secteur culturel.

33. D'autres sous-champs (religieux, écologiste, universitaire, lesbien) aurait pu être retenus. Encore une fois, il a fallu circonscrire étroitement notre objet. Des limites d'espace nous ont également contraint à réduire le nombre d'organismes considérés pour chacun des sous-champs. Nous avons choisi de mettre l'accent sur le sous-champ politicoadministratif, à défaut de pouvoir couvrir convenablement l'ensemble du champ. 


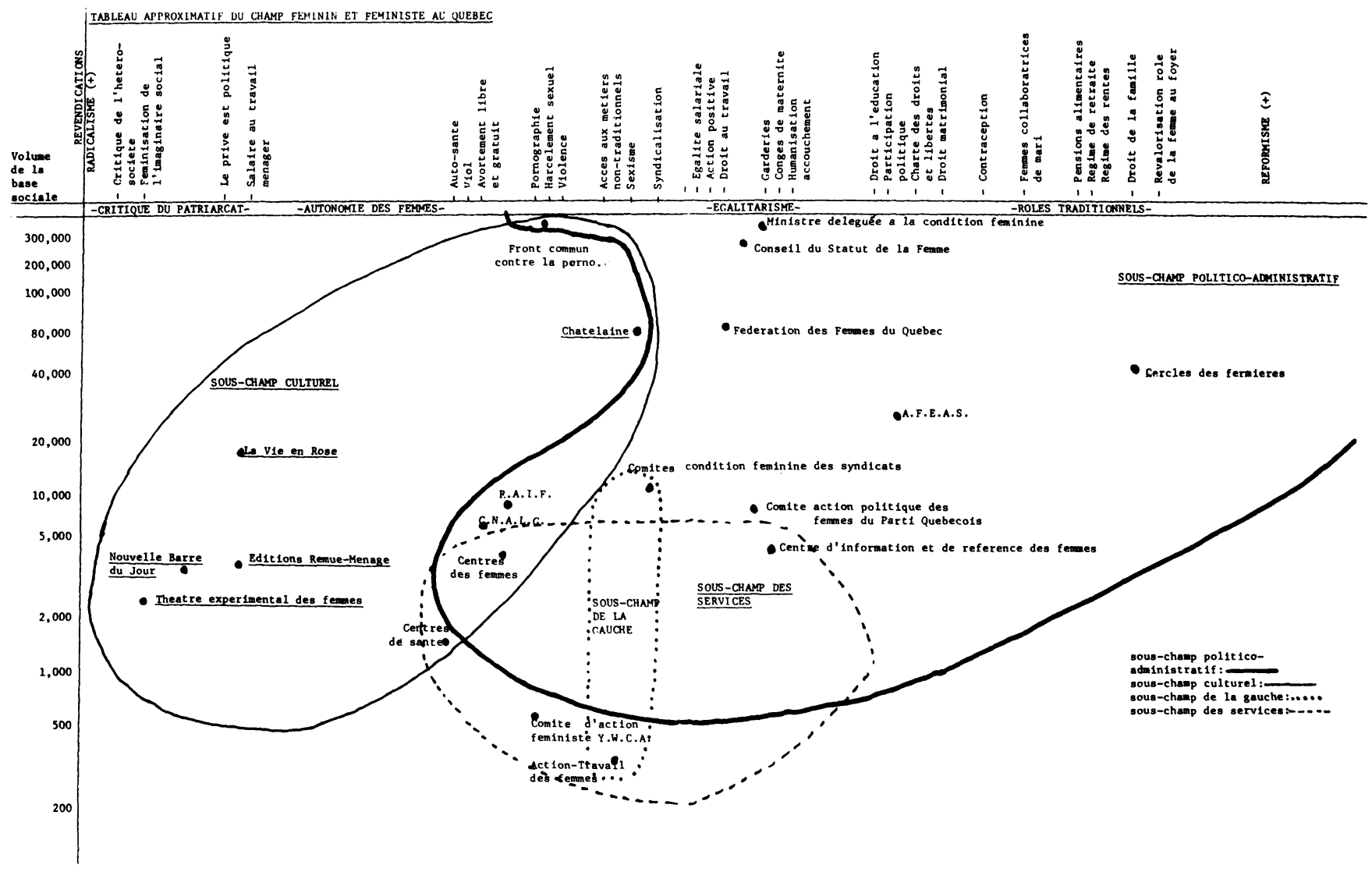


champ. Nous procèderons en présentant un à un les sous-champs, ainsi que les organisations qui y interviennent.

\section{Le secteur politico-administratif}

Le mouvement des femmes est à la fois un mouvement de revendication et de transformation sociale. Une part importante des actions entreprises sont dirigées vers l'État (lobbying pour l'implantation de nouvelles politiques, pour la mise en place de services, revendications relatives à des transformations juridiques etc.). Il n'est donc guère surprenant qu'un bon nombre d'organisations prennent place dans le secteur politico-administratif. C'est notamment le cas des institutions gouvernementales (Conseil du Statut de la Femme) et politiques (Ministre déléguée à la condition féminine, Comité d'action politique des Femmes du Parti Québécois), des associations dont l'action repose sur le lobbying (Fédération des Femmes du Québec, A.F.E.A.S., R.A.I.F.), et des groupes de pression ( «single issue movements»: Coordination nationale pour l'avortement libre et gratuit, Front commun contre la pornographie). On verra que ce sous-champ occupe une position dominante dans le champ féminin/féministe.

Le poste de Ministre déléguée à la condition féminine occupe une position dominante dans le champ. Son intégration à l'appareil politique détermine sa position ${ }^{34}$. Elle lui procure légitimité, infrastructure administrative, ressources financières. En outre, ce poste est la courroie nécessaire entre les revendications du mouvement et leurs réalisations. Ceci confere à la Ministre une influence sur les groupes, ne serait-ce que par la sélection des revendications qu'elle exerce dans le processus d'allocation des ressources.

34. L'inclusion de ce poste dans le secteur politico-administratif du champ féminin/féministe n'exclut pas sa participation au champ politico-administratif. Ici, l'analyse se doit de rendre compte de la multiplicité des identités sociales des organisations. 
Les interventions de la Ministre la positionne dans le pôle réformiste du champ. Son mandat consiste à réaliser le livre blanc

Pour les Québécoises, égalité et indépendance.

Le Conseil du Statut de la Femme occupe également une position dominante dans le champ. Il dispose d'un budget de loin supérieur à celui des autres organisations du champ ${ }^{35}$. Sa stabilité lui permet d'assurer une continuité essentielle dans cet espace social où les organismes ont souvent la vie courte. Cette même stabilité, ainsi que la présence d'une équipe de recherche, lui procurent une expertise importante. En outre, le Conseil est bien implanté en région, à travers le réseau Consult-Action, ce qui confere une certaine légitimité à ses actions. Donc, tant au niveau économique et organisationnel qu'aux niveaux scientifique et symbolique, le Conseil est doté de bases objectives d'influence importantes.

La position du Conseil sur la situation des femmes est résumée dans Pour les Québécoises, égalité et indépendance. Cette position est essentiellement égalitariste: droit à l'éducation, intégration économique des femmes, égalité juridique, lutte contre le sexisme etc... Il reprend surtout les revendications des organismes dont il reçoit le plus de soutien, soit L'A.F.E.A.S. et la F.F.Q. ${ }^{36}$.

Les groupes autonomes de femmes s'opposent au Conseil à certains égards ${ }^{37}$. Ils lui reprochent sa tiédeur, l'absence d'une

35. En 1980, le budget du Conseil était de $\$ 1950200$. En 1983 , il reçoit des crédits budgétaires de $\$ 2713400$.

36. Le Conseil occupe donc une position dominante dans le pôle réformiste du champ. Ce commentaire reflète précisément cette position: «Le C.S.F. a contribué à donner au féminisme québécois ses «lettres de noblesses". Mais le C.S.F., c'est aussi le pouvoir (...), c'est le féminisme d'État.» O'Leary, V., Toupin, L., Québécoises Deboute:, Montréal, Éditions Remue-Ménage, 1982, 14.

37. Par "groupes autonomes de femmes", on désigne des organismes du pôle radical du chamlp féminin/féministe. Parmi ceux ayant la plus grande visibilité, mentionnons La Vie en Rose, les Éditions Remue-Ménage, la Coordination nationale pour l'Avortement libre et gratuit, le Mouvement contre le Viol. 
remise en question des rapports de sexe, son support implicite à l'intégration des femmes aux structures patriarcales. Ses interventions sont senties comme parole usurpée, comme appropriation illégitime du mouvement ${ }^{38}$. On le critique pour ses structures bureaucratiques, pour ses plantes vertes. Ces conflits sont le reflet de la distance sociale entre le Conseil et les groupes autonomes, du fait qu'ils fonctionnent dans des espaces sociaux où les lois et les critères de légitimité different ${ }^{39}$. Ils sont aussi indice de l'important écart entre les positions hiérarchiques des deux types d'institutions dans le champ. En luttant contre la récupération, les groupes autonomes cherchent à légitimer le seul type d'action qu'ils soient objectivement en mesure d'entreprendre, compte tenu des moyens dont ils disposent.

La Fédération des Femmes du Québec (F.F.Q.) est l'association féminine qui occupe traditionnellement la position la plus élevée dans le champ féminin/féministe. Fondée en 1966, il s'agit d'une des plus vieilles institutions du champ, ce qui lui procure une certaine légitimité. Elle regroupe de nombreuses associations et représente au total 75000 membres. Elle est très proche du Conseil du Statut de la Femme, ayant été à l'origine de sa création en 1973. Elle détient donc principalement une base sociale et symbolique d'influence, ainsi que des alliances institutionnelles importantes. La Fédération traverse cependant une crise interne

38. "Tout ce que peut dire le C.S.F., c'est qu'il promet des réformes à la suite de consultations auprès de maints groupes et organismes existants, réformes qui, si elles sont prises en considération par le gouvernement, tendront à amoindrir les inégalités entre les hommes et les femmes. C'est vraiment le seul mandat dont le C.S.F. peut se réclamer. Et d'aucun autre. Le mandat de représenter les «féministes» (ou de se faire prendre pour toutes les féministes) (...) ne lui a jamais été donné explicitement, sauf peut-être par les groupes consultés." Ibid, 16.

39. Des différences relatives aux modalités d'interaction interpersonnelles sont indicatives de la distance sociale entre les deux types d'institution. Au sein des groupes autonomes, les rapports interpersonnels sont moins institutionnalisés. On nous faisait remarquer en entrevue que ces rapports fonctionnent sur le modèle familial, basés sur des jeux d'influence, sur le non-dit. 
majeure: chute du membership, problèmes de représentativité interne, crise de leadership. Ces facteurs contribuent à diminuer son influence traditionnelle dans le champ ${ }^{40}$.

L'objectif principal de la Fédération est de travailler à «atteindre l'égalité et la pleine participation des femmes au développement du Québec», en "permettant aux femmes de s'exprimer avec plus de poids» ${ }^{41}$. Les domaines d'action sont les suivants: éducation (sexisme, retour aux études), maternité désirée (avortement, contraception, éducation sexuelle, garderies, congés de maternité), la santé, le loisir, le travail (action positive, accès aux métiers non traditionnels) et la sécurité économique (régime des rentes, pensions, revenu minimum garanti...). La Fédération se positionne au centre du pôle réformiste, la problématique sousjacente à ses interventions étant nettement égalitariste.

Les Cercles des Fermières occupent également une position élevée dans le champ, principalement à cause de l'importance de sa base sociale (74387 membres en 1983). Leur influence est cependant moins importante que celle de la F.F.Q.: d'abord, leur intervention dans le champ féminin/féministe demeure limitée. Les Cercles agissent surtout comme club social et ne travaillent que sur un nombre réduit d'enjeux (politique de la famille, régime des rentes, restructuration scolaire). Ensuite, les Cercles soutiennent explicitement le maintien des fonctions féminines traditionnelles; ${ }^{42}$

40. Cette crise s'objectifie dans le choix de faire du congrès 1983 un congrès de réorientation. Elle traduit une transformation du rôle social de la Fédération. En 1966, il s'agit d'un regroupement de professionnelles (et d'épouses de professionnelles) œuvrant à la promotion de la femme. Les membres travaillent essentiellement à augmenter la participation politique des femmes, ce qui suppose un travail de propagation sociale de la socialisation politique légitime, la leur. Influencé par le développement du mouvement des femmes, la Fédération tend maintenant à devenir davantage un mouvement de revendication. 1982,4 .

41. La Petite Presse (Bulletin d'information de la F.F.Q.), $2^{c}$ année, no. 4, octobre

42. Leurs objectifs consistent entre autres à «transmettre le patrimoine» et à faire «l'enseignement et la promotion des arts-ménagers», ce qui correspond bien aux fonctions traditionnelles des femmes. 
ils vont à l'encontre des tendances dominantes du champ, ce qui réduit leur influence potentiellement soutenue par l'importance de leur membership. Enfin, les membres ne détiennent pas les attributs sociaux légitimes du champ. ${ }^{43} 72 \%$ d'entre elles sont reines du foyer, $83 \%$ catholiques pratiquantes, de milieu rural et semi-urbain. Ces attributs sont des stigmates qui les marginalisent dans le champ et limitent leur influence. ${ }^{44}$

L'Association féminine pour l'Éducation et l'Action sociale (A.F.E.A.S.) exerce dans le champ une influence supérieure à celle qui serait soutenue par la seule force de son membership. Regroupant 35,000 membres, l'association a mis en place des mécanismes de consultation exceptionnellement bien rodés, ce qui confere une grande légitimité à ses prises de positions. ${ }^{45}$ À partir de dossiers très fouillés, les membres font du lobbying au niveau national, débordant le champ politico-administratif, pénétrant la société civile. ${ }^{46}$ Ces facteurs contribuent à renforcer sa position dans le champ.

43. Les attributs sociaux légitimes du champ correspondraient aux attributs détenus par les agents qui y occupent des positions dominantes: moyennement scolarisées, professionnelles, provenant d'un milieu urbain, revenus de $\$ 25000$ et plus, «intéressée, ouverte, curieuse, active, dynamique, engagée».

44. Les membres de l'A.F.E.A.S. ont des caractéristiques similaires, quoiqu'en des proportions moindres.

45. Les 600 associations locales se rencontrent mensuellement pour discuter des divers dossiers. Les propositions émises sont éventuellement acheminées à l'assemblée générale annuelle, regroupant 1000 déléguées. L'A.F.E.A.S. peut ainsi transmettre réellement la volonté de la majorité des membres. Il s'agit de la seule association féminine à avoir été invitée à participer au dernier sommet économique québécois, ce qui est indice de la légitimité sociale qu'elle détient, et de sa proximité aux institutions sociales dominantes.

46. Leur lobbying s'effectue non seulement auprès des organismes gouvernementaux, mais aussi auprès des chambres de commerces, des clubs sociaux (Richelieu, Rotary), des députés, des maires... 
Mouvement d'inspiration chrétienne, l'A.F.E.A.S. cherche à lier l'ancien et le moderne: elle travaille présentement à la valorisation de la femme au foyer, afin que celle-ci soit reconnue comme citoyenne à part entière ${ }^{47}$. Ses autres dossiers majeurs femmes collaboratrices de maris, accès à l'éducation - s'inscrivent dans une stratégie visant à promouvoir les femmes exerçant des fonctions traditionnelles.

Les moyens d'action adoptés par l'A.F.E.A.S. sont indicatifs de sa position dans le spectre polaire: proche collaboratrice du Conseil du Statut de la Femme, elle adopte des modes d'intervention institutionnels, ce qui suppose une certaine proximité sociale au champ politico-administratif, ainsi qu'une conception similaire du type d'intervention politique légitime ${ }^{48}$.

Le Front commun contre la Pornographie regroupe 300000 membres répartis dans 70 associations. Il s'agit d'un des groupes de pression ayant actuellement la plus grande visibilité dans le champ. La question de la pornographie réunit dans un même front tant les organismes radicaux que les organismes réformistes. $\mathrm{Y}$ voisinent les arguments anti-patriarcaux et les arguments puritains $^{49}$. La loi 109 sur le cinéma et le vidéo, ainsi que les clubs érotiques constituent leur fer de lance.

Sans nier le fait que le Front constitue le point de convergence le plus important du mouvement actuellement, on pourrait penser que sa position est conjoncturelle: son influence repose entre autres

47. Ce témoignage est illustratif du type d'action valorisé par l'A.F.E.A.S.: «Je reste membre de l'A.F.E.A.S., femme engagée au Conseil d'administration. Je suis membre active bénévole à temps plein, et engagée, mais surtout, mère de famille. "Femmes d'Ici (Bulletin d'information de l'A.F.E.A.S.), vol. 17.

48. Louise Joly, vice-présidente de l'A.F.E.A.S., précise les positions de l'organisation: «Oui, on est féministe, parce qu'on s'occupe de la condition de la femme, des choses féminines. On n'est pas radicales, ou extrémistes. On ne porte pas de pancartes, on ne donne pas de coups de poings sur la table. On y va plus modéré, on s'arme de patience. On ne porte pas de bannières."

49. D'où le fait que le Front ait été situé au centre du spectre polaire. 
sur des alliances entre organismes diamétralement opposés dans le champ.

Le R.A.I.F. (Réseau d'action et d'information pour les femmes) occupe une position hiérarchique moyenne. Dès 1973, il articule un discours féministe «recevable» dans le champ politique. Passablement radical ${ }^{50}$, il ne remet pas en question la légitimité des appareils gouvernementaux ${ }^{51}$. Il parvient ainsi à occuper une position centrale dans le champ. Il y acquiert une grande visibilité, d'abord à cause des appuis dont il dispose dans les médias (Châtelaine, Le Soleil ${ }^{52}$, et ensuite, parce qu'il intervient stratégiquement à travers des déclarations publiques fracassantes. Ainsi, en dépit de la faiblesse relative de sa base sociale, il exerce une certaine influence dans le champ.

La Coordination nationale pour l'Avortement libre et gratuit (C.N.A.L.G.) occupe une position similaire à celle du R.A.I.F., mais à partir de bases d'influences différentes. Elle est héritière de la revendication qui a été la clef de voûte du féminisme radical entre 1975 et $1980^{53}$, ce qui lui confere une certaine légitimité.

50. Le R.A.I.F. a été très impliqué dans la bataille du nom légal. Il a également travaillé sur les garderies, l'avortement, le droit au travail, le sexisme et le revenu minimum garanti. Ses méthodes d'intervention institutionnelles nous interdisent de le classer au centre du pôle radical.

51. "On peut arracher la discrimination sans détruire, avec les outils du système lui-même, qui restent là, si souvent inutilisés. Il ne s'agit que de plonger dans les lois sans crainte, de feuilleter les projets qu'on nous prépare, avec un sens critique et féministe, exigeant sans lâcher prise, les changements nécessaires." R.A.I.F., revue féministe, no. 6768 , juillet 1981,15 .

52. En 1981, il dénonce à grands bruits la violence du pape contre les femmes et les enfants. Ses interventions auprès de diverses commissions parlementaires sont très remarquées.

53. Entre 1974 et 1978 , le Comité de Lutte pour l'Avortement et la Contraception libres et gratuits (C.L.A.C.L.G.) parvient, avec l'appui des syndicats, à créer une mobilisation importante autour de l'avortement. C'est autour de cette question, et de celle du salaire au travail ménager, que se réunissent les institutions du pôle radical du champ (Théatre des Cuisines, Éditions Remue-Ménage, Centre de documentation féministe, Centre de Santé des femmes du Quartier Plateau Mont-Royal). Le 31 mars 1979 a lieu une manifestation de 2500 personnes pour la Journée Internationale de l'Avortement. Voir Lamoureux, D., "La lutte pour le droit à l'avortement au Québec, 1969-81", Revue d'bistoire de l'Amérique française, juin 1983. 
Elle dispose d'alliances institutionnelles importantes, particulièrement dans le pôle radical du champ, et auprès des syndicats.

Présentement, la C.N.A.L.G. s'efface devant le Front commun contre la Pornographie. La revendication de l'avortement est mise en veilleuse, entre autres par volonté de protéger les acquis (Cliniques Lazure, centres de santé où sont pratiqués des avortements défrayés par l'assurance-maladie). Pour les militantes, le Front commun contre la Pornographie devient le lieu privilégié pour faire avancer le mouvement.

Organisme exerçant des pressions politiques pour la mise en place de services, la Coordination se situe à l'intersection du sous-champ des services et du sous-champ politico-administratif. Son travail de sensibilisation l'inscrit dans le sous-champ culturel.

Le Comité d'action politique des femmes du Parti Québécois occupe également une position hiérarchique moyenne dans le champ féminin/féministe. Son intégration au parti sous-tend potentiellement une influence importante. Cependant, le comité manque de légitimité proprement féministe. Il est perçu comme une «succursale» du pouvoir, ayant les mains liées aux décisions du congrès ${ }^{54}$. Son influence sur le champ est ainsi limitée ${ }^{55}$. En outre, le mouvement de démobilisation qui traverse le parti, ainsi que le désenchantement des militantes (provoqué par leur quasi-exclusion lors de la distribution des fruits de la victoire en 1976) affaiblissent sa position.

Ce comité se situe dans le pôle réformiste du champ féminin/ féministe. Ne remettant pas en question les structures familiales traditionnelles, il fait endosser au congrès de 1977 les congés de

54. "Comme on est militantes à l'intérieur du Parti, il faut que l'on soit solidaire. On ne peut pas se prononcer contre ce qui a été voté au congrès. Ça veut dire qu'on discute avec le Parti avant de faire du «chiard» (sic) public.» Claudette Bastien.

55. Ce n'est qu'en 1977 que les femmes du Parti Québécois se mobilisent vraiment en tant que groupe, se situant dans l'aile progressiste du Parti, avec leurs propositions concernant l'avortement. Voir "Quand les femmes du Oui disent Non", La Vie en Rose, mars-avril-mai 1981, 28. 
maternité, les garderies, l'élimination de la discrimination au travail $^{56}$. Ses prises de position relatives à l'avortement le ramènent près du pôle radical mais ces tendances sont atténuées par la tiédeur des positions du parti considéré dans son ensemble ${ }^{57}$.

Le secteur politico-administratif rassemble la majorité des organisations occupant des positions élevées dans le champ féminin/ féministe. La plupart d'entre elles ont une base sociale importante, comparativement aux autres organisations du champ. C'est là que l'on retrouve les organismes les plus anciens, ceux pour lesquels l'épreuve du temps procure une légitimité. En outre, la plupart de ces institutions ont recours à des moyens d'intervention politique institutionnels (présentation de mémoire, rencontre de ministres, lobbying). Ceci confirme leur proximité sociale avec le champ politico-administratif, et est indice de leur position élevée dans le champ, par association aux institutions sociales dominantes.

Ces organisations se situent majoritairement dans le pôle réformiste du champ. Ne questionnant pas la légitimité des appareils politiques, elles adaptent leurs revendications au possible immédiat, ce qui en fait des interlocuteurs audibles.

\section{Le secteur de la gauche}

C'est à la fin des années ' 60 que le mouvement des femmes trouve son deuxième souffle, après la bataille des suffragettes de la première moitié du $20^{\mathrm{e}}$ siècle. Dans un tel contexte, il n'est guère surprenant que le mouvement des femmes soit forcé de se définir par rapport aux autres courants sociaux de l'époque, et particulièrement, par rapport au mouvement ouvrier. Issues souvent de la gauche, les militantes devront reformuler les problématiques

56. Le Parti Québécois met l'emphase sur la politique familiale et les garderies, ainsi que sur une politique nataliste (voir le programme 1978).

57. Rappelons que le Parti Québécois a refusé d'endosser le Manifeste des Femmes Québécoises, lequel adoptait une perspective relativement radicale (Voir Tremblay, G., "Manifeste des Femmes Québécoises», Possibles, vol. 5, no. 3-4, 1981). 
de manière à justifier théoriquement l'émergence d'une lutte des femmes, et à contrer sa secondarisation par rapport à la lutte des classes $^{58}$.

Si les institutions de ce secteur se situent à la gauche du spectre politique, leur attitude par rapport aux problématiques féministes les situent plutôt dans le pôle réformiste du champ féminin/féministe. Leurs prises de position sont souvent circonscrites par la défense du droit des femmes au travail.

Ce secteur occupe une position plus basse que le secteur politico-administratif ${ }^{39}$. Les institutions qu'il regroupe ont surtout recours à des moyens d'intervention non-institutionnels, fondés sur la mobilisation de leur base sociale (manifestation, pétition, rassemblement). Leur influence dans le champ demeure limitée par la nécessaire conciliation de leurs actions avec les enjeux propres au champ de la gauche.

58. Il est important d'isoler ce sous-champ dans l'analyse pour rendre compte de l'évolution du mouvement des femmes au cours des années 70. La renaissance du mouvement féministe passe par son autonomisation institutionnelle à l'égard de la gauche. Il s'agit de faire reconnaître la légitimité du mouvement des femmes. Le Front de Libération des Femmes du Québec (1969-71), le Centre des Femmes (1972-75), le Comité de Lutte pour l'Avortement et la Contraception libres et gratuits (1974-81), la revue Québécoise deboute! (1971-74) seront au centre de ce mouvement d'autonomisation. Cette lutte se soldera finalement par la dislocation du groupe En Lutte! en 1982, précisément autour de la question du rapport lutte des classes/luttes des femmes (Voir Lamoureux, J., Lutte des femmes et socialisme, combat difficile. possible, urgent (discours d'En Lutte, journée internationale de la femme 1981), document ronéotypé.

Louise Toupin dresse un portrait des luttes de l'époque: «D'abord membres à part entière de cette nouvelle gauche progressiste, quoique regardés avec une certaine suspicion parce que "féministes", le Centre des Femmes, puis le Théâtre des Cuisines et d'autres groupes de cette tendance féministe "marxiste» deviendront des "ennemis de la classe ouvrière" (...). Parce que les féministes refusent de faire de la lutte des femmes un simple contradiction secondaire du capitalisme, elles seront accusées par les groupes "m- 1 " de créer une division "au sein des masses" entre les hommes et les femmes." Québeioisises Deboute!, op. cit., 35. C'est de son autonomisation à l'égard de la gauche que le pôle radical du mouvement féministe tirera son appellation «mouvement autonome des femmes".

59. Ce sous-champ peut être compris comme extension du champ politicoadministratif. Ses institutions (syndicats, groupes populaires) redétinissent le social de manière à faire passer le politique, de sa dimension institutionnelle traditionnelle (partis. gouvernement, État) aux rapports de classe dans la société civile. 
Les Comités de la condition féminine des syndicats (C.S.N., F.T.Q., C.E.Q.) occupent les positions dominantes du sous-champ de la gauche. Leur intégration aux appareils syndicaux leur procure une base organisationnelle d'influence déterminante de leur positionnement. Elle leur fournit entre autres une infrastructure institutionnelle, une légitimité, et leur confere un savoir-faire politique que seule produit une socialisation au sein de grandes organisations.

Pour ces mêmes raisons, ces comités occupent une position médiane dans le champ féminin/féministe. Les alliances institutionnelle dont ces comités disposent dans le pôle radical du champ contrebalancent la relative faiblesse de leur base sociale d'influence. Leur position hiérarchique se reflète dans le fait que le Comité intersyndical à la condition féminine puisse prendre en charge l'organisation de la Journée du 8 mars.

Les Comités de la condition féminine prennent place au centre du spectre de polarisation. S'intéressant dans un premier temps aux revendications touchant les travailleuses (droit au travail, égalité salariale, action positive, garderies, congés de maternité), elles se radicalisent dans un deuxième temps, se rapprochant du mouvement autonome des femmes sur la question du harcèlement sexuel et de l'avortement (particulièrement à la C.S.N.). Elles refuseront toutefois de supporter le salaire au travail ménager, celui-ci allant à l'encontre de l'accès des femmes au marché du travail. Leur double allégeance - mouvement syndical, mouvement des femmes - les place parfois dans une situation ambiguë ${ }^{60}$.

Action-Travail des femmes est un groupe populaire qui œuvre auprès des chômeuses et travailleuses non-qualifiées. L'accès aux métiers non-traditionnels, les transformations technologiques et le harcèlement sexuel sont ses principaux domaines d'intervention.

Cet organisme occupe une position basse dans le champ. En atteste la précarité de sa base économique d'influence, réduite mixte.

60. Ceci se reflète dans leur volonté de faire de la Journée du 8 mars une fête 
à des subventions gouvernementales toujours renouvelables. En outre, travaillant surtout sur des cas individuels, l'impact de son action demeure limité. Le même phénomène se produit au sein des institutions du sous-champ des services.

\section{Le secteur des services}

Considérées individuellement, les organisations du secteur des services ont une influence limitée dans le champ. D'abord, elles sont généralement organisées autour d'un seul type de service (aide aux femmes battues, garderies, avortement), ce qui tend à réduire leur rayonnement. Ensuite, la portée de leur action demeure limitée structurellement du fait que leurs interventions répondent à une multitude de besoins individuels. Par opposition, les associations féminines ont plus de visibilité, agissant dans l'énonciation des principes plutôt que dans leur application concrète. Enfin, les institutions de ce sous-champ ne détiennent généralement pas la base sociale nécessaire pour se prononcer avec une efficace politique sur les grands enjeux du champ.

Considérées globalement, les institutions du secteur des services ont toutefois un poids déterminant dans le champ féminin/ féministe. S'y regroupent une multitude d'organismes dynamiques rassemblant une large proportion de la base active du mouvement. Les femmes y travaillent quotidiennement, infinitésimalement, faisant de la conscientisation à long terme à partir d'interventions ponctuelles.

La problématique dominante au sein du sous-champ des services est celle de l'autonomie des femmes. Autonomie personnelle d'abord, au niveau financier, à l'égard des hommes et de l'État. Prise en main de leur destinée, capacité d'auto-détermination, de liberté. Le discours s'y fait plus ou moins radical selon les organisations et les agents qui s'y retrouvent, même si leurs revendications spécifiques les positionnent principalement dans le pôle 
réformiste du champ. Les interventions de ces institutions peuvent être comprises comme éléments d'un mouvement social autogestionnaire, visant la réappropriation par la société civile (ici par les femmes) de fonctions exercées par l'État (ici, dans le secteur des services).

Le Centre d'information et de référence pour Femmes est l'organisation dominante de ce sous-champ. Il emploie 15 permanentes et plus de 300 bénévoles y travaillent de façon régulière. Disposant d'un budget de $\$ 400000$ (1982), il diversifie ses sources de financement, contrebalançant ainsi le poids de ses diverses dépendances. Fondé en 1973, il détient la légitimité que lui confere son ancienneté dans le champ. Son fichier d'information est un rouage essentiel au fonctionnement du champ. En outre, son travail auprès des immigrantes lui a acquis une expertise reconnue par les instances gouvernementales.

Le Centre effectue essentiellement un travail d'information et de formation, assistant les femmes dans leurs démarches auprès des organismes gouvernementaux, les préparant à s'insérer sur le marché du travail, les aidant à acquérir une indépendance financière. Il intervient sporadiquement dans le champ politico-administratif, par la présentation de mémoires sur des questions ponctuelles (politique familiale, loi de l'immigration, pornographie etc.).

C'est au sein des Centres de femmes que se retrouve l'essentiel de la base active du mouvement des femmes. Ils occupent individuellement des positions peu élevées dans le champ ${ }^{61}$. Cependant, comme phénomène global, leur influence est considérable. De 4 qu'ils étaient en 1980, ils sont maintenant au nombre de 36.

61. Ces centres sont dans une situation financière précaire. $75 \%$ d'entre eux reçoivent des subventions des programmes fédéraux de création d'emploi et de développement communautaire, ou encore, du Secrétariat d'État. "Chacun demande des subventions, on passe notre temps dans la paperasse, à faire des rapports d'activité. Il faudrait trouver un moyen pour être moins dépendants. Le Ministère des Affaires Sociales a reçu cette année pour 60 millions de demandes de subventions, alors qu'il n'a que 5 millions à distribuer.» Une militante. 
Ces centres agissent en quelque sorte comme succursales locales du mouvement des femmes à travers le Québec. Ancrés dans les communautés, ils sont appelés à répondre à toutes sortes de demandes (assistance aux femmes battues, viol, avortement, auto-santé, harcèlement etc.). Selon les cas, ils appuient plus ou moins fortement le Front commun contre la Pornographie, la Coordination nationale pour l'Avortement libre et gratuit, le Mouvement contre le Viol. Ils participent activement à l'organisation du 8 mars en région. La problématique générale qui sous-tend leur action est celle de l'autonomie des femmes.

Les Centres de Santé sont proches des Centres de femmes. À partir d'une critique radicale des pratiques médicales traditionnelles, on y amène les femmes à prendre le contrôle de leur corps, tant au niveau de la gynécologie que par des pratiques d'avortement. Leur intervention s'inscrit dans le mouvement autonomiste. Ils sont présentement au nombre de 7 .

Le Comité d'action féministe du Y.W.C.A. travaille également à amener les femmes à se prendre en charge. La création du service La riposte des femmes, mouvement pour contrer la victimisation va dans ce sens. Ce comité occupe une position moyenne dans le souschamp des services. Son intégration au sein du Y.W.C.A. lui procure une base organisationnelle importante qui pallie à la faiblesse relative de sa base sociale.

Le radicalisme des organisations du sous-champ des services est souvent tempéré par les impératifs de l'action. Tel n'est pas le cas pour les institutions du sous-champ culturel, le domaine du symbolique laissant plus de place à l'utopie.

\section{Le secteur culturel}

Le secteur culturel regroupe des organisations qui ont pour objectif de transformer l'imaginaire social en le féminisant, en 
dénonçant les inégalités et les rapports de domination, et en y diffusant de nouvelles représentations sociales des femmes.

Au cours de la dernière décennie, ce sous-champ a été animé d'un grand dynamisme. Les revues se sont succédé62, agissant comme point de ralliement et centre de propagation du féminisme dans un moment où le mouvement autonome des femmes est particulièrement diffus et faiblement institutionnalisé. Il est vrai que la production symbolique est souvent moins onéreuse que la mise en place de structures institutionnelles. Il n'est donc guère surprenant que ce type d'intervention soit particulièrement présent au moment où le mouvement est dans ce processus de constitution.

Les organisations du secteur culturel se situent nettement dans le pôle radical du champ féminin/féministe. C'est là le terrain de la critique des structures patriarcales.

La revue $L a$ Vie en Rose est la principale instance de diffusion du pôle radical. Elle est là pour dénoncer alors que les autres revendiquent. Il s'agit de "concrétiser la solidarité des femmes en passant par l'information", "d'aider les femmes à devenir ce qu'elles sont, une force politique», et de «se prononcer sur tout ce qui peut donner une prise sur le réel aux femmes». La perspective adoptée est donc celle d'un journalisme de combat ${ }^{63}$.

62. Les principales revues ont été Québécoises Deboute! (1970-75, réunissant des féministes marxistes), Les Têtes de Pioche (1976-79, première revue radicale en rupture avec le féminisme marxiste), Pluri-Elles (1977-79), et Des Luttes et des Rires de Femmes (1979-81, voulant agir dans un premier temps comme centre de regroupement des diverses tendances du mouvement féministe autonome). La Vie en Rose (fondée en 1980) hérite du travail effectué par ces divers collectifs au cours des années 70 .

63. Marque de leur position dans le milieu des médias, des membres du collectif attaque l'objectivité journalistique en l'associant à la défense d'intérêts corporatistes: «Troublée par la méfiance d'autres femmes journalistes qui, en se démarquant des groupes de femmes et de leurs demandes, en réitérant la primauté de leurs critères professionnels et de la spécificité de cette profession, me semblent défendre leurs privilèges plutôt que le droit du public à l'information, la crédibilité journalistique et toutes ces faciles justifications du non-engagement». Thérèse, Janette, Hélène et les autres, ou la face cachée de l'information, op. cit., 46. 
La revue est mise sur pied en 1980 par un groupe de femmes issues majoritairement du Comité de Lutte pour l'Avortement et la Contraception libres et gratuits. Ces femmes ont derrière elles une longue militance dans les organisations radicales du champ ${ }^{64}$. Elles en ont profondément incorporé l'ethos et peuvent ainsi transmettre avec beaucoup d'humour le discours féministe radical.

La Vie en Rose occupe une position nettement dominante dans le sous-champ culturel. Tirant à 20000 exemplaires, elle peut déborder les cercles clos des militantes assidues pour toucher le grand public et donner une visibilité sans précédent au féminisme radical. En outre, elle dispose d'alliances institutionnelles importantes dans les sous-champs de la gauche, des services et dans le sous-champ culturel ${ }^{65}$.

Chatelaine est le pendant réformiste de La Vie en Rose. Cette revue occupe une position élevée dans le champ culturel québécois, tirant à 295000 exemplaires. Ceci lui permet de conserver une position élevée dans le champ féminin/féministe, malgré que certains la considèrent par trop "féminine» ${ }^{66}$.

L'effet produit par cette revue relativement aux enjeux du champ n'est pas à négliger. Subrepticement, à long terme, elle diffuse de nouvelles représentations sociales des femmes qui s'in-

64. Mentionnons leur proximité avec le champ contre-culturel québécois, cristallisé dans l'intégration de La Vie en Rose des débuts à la revue Le temps fou.

65. La revue ne prétend pas être le porte-parole du féminisme radical. «L'autonomie signifie que nous travaillons d'abord pour nous-même, à partir de notre réalité, sans avoir à justifier nos intérêts, nos priorités, nos choix. Liberté d'esprit. Nous ignorons le spectre des intérêts supérieurs." La Vie en Rose, mars-avril-mai 1981, 3. Revue autonome donc, elle cherche toutefois à être au service des groupes de femmes, ce qui renforce sa position dans le champ.

66. «Au mieux, il s'agit d'un réformisme classique visant à améliorer l'adaptation des femmes aux structures déjà existantes. La publication du dossier sur l'avortement, sur les femmes et leur gynécologue avait fait croire à un vent de libéralisme..." La Vie en Rose, sept.-oct.-nov. 1981, 27. 
corporent à la culture dominante et contribuent à promouvoir les transformations qui sont les enjeux du champ ${ }^{67}$.

La Nouvelle Barre du Jour s'inscrit dans le champ féminin/ féministe à cause du rôle qu'elle a joué dans l'implantation d'une écriture féministe au Québec. Cette revue occupe une position élevée dans le champ littéraire québécois. Associée au courant de la Nouvelle Écriture, elle cherche à révolutionner les formes d'expression et se situe ainsi à l'avant-garde de la création littéraire ${ }^{68}$. Sa fondatrice, Nicole Brossard, occupe une position nettement dominante dans ce champ ${ }^{69}$.

Suivant les normes de production propres au champ symbolique où elle s'inscrit, la revue ne peut rencontrer les schèmes de lecture dominants. Il existe donc une importante distance culturelle entre la revue et le champ féminin/féministe, laquelle se manifeste dans des difficultés d'appropriation des œuvres par certaines militantes ayant moins de proximité à une culture scolaire «de distinction» ${ }^{70}$.

À l'intérieur du champ féminin/féministe, La Nouvelle Barre $d u$ Jour occupe une position moyenne. Si son influence demeure limitée par l'hermétisme des formes d'expression produites, elle est renforcée du fait de sa position dans le champ littéraire (prestige), et du fait de l'importante visibilité dont jouissent les écrivaines féministes au Québec.

67. Ceci soulève la question des fonctions sociales de la littérature féminine, dont nous ne pouvons discuter ici.

68. La Nouvelle Barre du Jour, cherche à déclasser dans le champ littéraire québécois la revue Liberté plus traditionnelle.

69. En atteste le fait qu'ait été organisé à l'U.Q.A.M. cette année un colloque de 10 jours autour de son œuvre.

70. Cette distance est illustrée clairement dans cet extrait: «Si l'écriture est très belle, finement ciselée, découpée et fort savante, le sens par contre est souvent fuyant et confident (...). D'où la difficulté énorme que j'éprouve à parler de ce livre, l'émotion et la signification étant constamment filtrées par la forme de l'écriture et donc constamment perrçue avec une distance qui ne permet aucune participation directe et sensible au processus émotif et réflexif de ces femmes.» La Vie en Rose, juin-juillet-août 1982, 68. 
Le Théâtre Expérimental des Femmes occupe une position similaire à celle de la Nouvelle Barre du Jour dans le champ, effectuant un travail similaire à partir d'un médium culturel autre. On cherche à révolutionner l'expression et les formes pour les faire correspondre à une vision féministe de la réalité. Le thème du lesbiannisme revient couramment dans ses productions. Cette organisation occupe une position médiane dans le champ féminin/féministe, principalement à cause de la légitimité qu'elle détient dans le champ théâtral.

Les Éditions Remue-Ménage est la principale maison d'édition du pôle radical du champ. Elle y occupe une position moyenne, contribuant à la visibilité des revendications et problématiques féministes. Sa fonction en fait un rouage essentiel au mouvement autonome des femmes, et la met en rapport constant avec diverses institutions radicales. La spécificité de son public lui permet de se tailler un créneau dans le champ littéraire.

Lors de sa création en 1976 , cette maison avait pour objectif de «reprendre le discours féministe pour ne pas le laisser aux groupes de gauche». Elle participe activement au mouvement d'autonomisation du féminisme radical. Dans ce contexte, le salaire au travail ménager devient son cheval de bataille.

Les organisations du secteur culturel tiennent surtout leur position de l'importance de leur base symbolique d'influence, la valeur de la production culturelle ne pouvant se mesurer qu'à la légitimité qu'elle détient. Elles s'attaquent à un des mécanismes essentiels de la reproduction des rapports de sexe, en travaillant sur la forme et le contenu des représentations sociales.

\section{CONCLUSION}

Dans les pages précédentes, nous avons construit partiellement le champ féminin et féministe québécois. Pour ce faire, nous avons mis en rapport certaines de ses organisations, de manière 
à dégager leurs propriétés spécifiques. Nous avons décrit les bases objectives sur lesquelles repose leur influence potentielle. Simultanément, nous justifions leur positionnement dans le tableau représentant le champ. Nous avons également situé ces organisations dans un axe radicalisme/réformisme renvoyant aux différences relatives à la définition des nouvelles fonctions, positions et représentations sociales des femmes.

Les principales caractéristiques dégagées pour les divers secteurs considérés sont les suivantes:

1- Le secteur politico-administratif occupe une position dominante dans le champ, réunissant les organisations ayant une base organisationnelle, symbolique et sociale d'influence supérieure. La plupart des organismes regroupés dans ce sous-champ sont de caractère réformiste. Ceci porte à penser que la position hiérarchique des organisations est généralement corrélée avec leur degré de proximité à la culture et aux institutions socialement dominantes.

2- Le secteur de gauche occupe une position peu élevée dans le champ. Les organisations qu'il regroupe doivent concilier leurs interventions dans le champ féminin/féministe avec les enjeux propres au champ de la gauche, ce qui réduit leur influence.

3- Le secteur des services occupe une position inférieure dans le champ féminin/féministe. Le caractère concret et appliqué du type d'intervention qui y est effectué réduit sa portée. Ces interventions ne partent pas de principes abstraits universalisables (par l'intermédiaire de lois), comme c'est le cas pour les interventions du sous-champ politico-administratif.

4- Les organisations du secteur culturel se situent nettement dans le pôle radical du champ: la confrontation du désirable et du possible y est moins violente. Les institutions de ce souschamp ont comme principale base d'influence, une base symbolique.

La prise en compte des sous-champs dans l'analyse voulait traduire le fait que le champ féminin/féministe fonctionne en interaction avec des espaces sociaux autres. On voulait ainsi éviter 
de poser abstraitement la question de l'autonomie relative de ce champ, cherchant plutôt à voir comment il fonctionne concrètement, dans la rencontre de divers enjeux.

Dans la mesure où l'analyse descriptive présentée ici est approximative et partielle, elle doit être lue comme instrument de recherche jetant les balises d'une compréhension politique du champ féminin/féministe. La construction des structures politiques du champ permet d'éclairer des relations invisibles pour quiconque adopte un point de vue «interniste». D'où la justification d'une telle approche, et son intérêt. 